\title{
Millennials e internet: cómo los estudiantes de Comunicación iberoamericanos utilizan y valoran las redes sociales
}

\author{
Santiago Tejedor \\ Ricardo Carniel Bugs \\ Santiago Giraldo Luque \\ Universitat Autònoma de Barcelona. Departament de Periodisme i de Ciències de la \\ Comunicació \\ santiago.tejedor@uab.cat \\ ricardo.carniel@uab.cat \\ santiago.giraldo@uab.cat
}

Fecha de presentación: julio de 2018

Fecha de aceptación: febrero de 2019

Fecha de publicación: junio de 2019

Cita recomendada: Tejedor, S.; CARniel Bugs, R. y GiRAldo LuQue, S. (2019). «Millennials e internet: cómo los estudiantes de Comunicación iberoamericanos utilizan y valoran las redes sociales». Anàlisi. Quaderns de Comunicació i Cultura, 60, 43-63. DOI: <https://doi. org/10.5565/rev/analisi.3167>

\section{Resumen}

El uso de las tecnologías por parte de la generación millennial se ha convertido en objeto de análisis de numerosos estudios. Este artículo presenta los resultados de una investigación realizada en 16 países iberoamericanos con 1.080 estudiantes universitarios de Comunicación que reflexionaron sobre la importancia y el uso que hacen de las redes sociales en sus relaciones sociales y en sus procesos informativos y comunicativos. El estudio, que reunió un total de 32.400 evidencias, identifica una tendencia a nivel internacional sobre los hábitos de información del alumnado entre las redes sociales y los sitios web de los cibermedios. También advierte de una confusión terminológica en la noción de los estudiantes sobre conceptos decisivos relacionados con las redes sociales y el nuevo escenario comunicativo digital.

Palabras clave: jóvenes; universitarios; comunicación; redes sociales; Iberoamérica

Resum. Mil.lennistes i internet: com els estudiants de Comunicació iberoamericans utilitzen $i$ valoren les xarxes socials

L'ús de les tecnologies per part de la generació mil.lennista s'ha convertit en objecte d'anàlisi de nombrosos estudis. Aquest article presenta els resultats d'una investigació realitzada 
en setze països iberoamericans amb 1.080 estudiants universitaris de Comunicació que van reflexionar sobre la importància i l'ús que fan de les xarxes socials en les seves relacions socials i en els seus processos informatius i comunicatius. L'estudi, que va reunir un total de 32.400 evidències, identifica una tendència internacional en els hàbits d'informació de l'alumnat entre les xarxes socials i els llocs web dels cibermitjans. També adverteix d'una confusió terminològica en la noció dels estudiants sobre conceptes decisius relacionats amb les xarxes socials i el nou escenari comunicatiu digital.

Paraules clau: joves; universitaris; comunicació; xarxes socials; Iberoamèrica

Abstract. Millennials and the Internet: How Ibero-American students of communication use and value social media

The use of technologies by the millennial generation has become the subject of analysis of numerous studies. This article presents the results of research carried out in 16 IberoAmerican countries with 1,080 university students of communication who reflected on the importance and use they make of social media in their social relations and in their informative and communicative processes. Based on 32,400 data items, the study identifies an international trend in the information habits of students in social media and cyber media websites. In addition, the article warns about a terminological confusion in the students' notion of decisive concepts related to social networks and the new digital communicative scenario.

Keywords: youth; university students; communication; social media; Ibero-America

\section{Introducción}

Las redes sociales han contribuido de forma intensa a la consolidación del nuevo entorno comunicativo (Castells, 2003a) y su crecimiento ha generado transformaciones en diferentes ámbitos de la sociedad (Barabási y Bonabeau, 2003). Se plantea, por tanto, la necesidad de redefinir y analizar, desde nuevos enfoques, las estructuras mediáticas - locales y globales - en el marco de unos contextos económicos, políticos, culturales y educativos que han adquirido una esencia líquida (Bauman, 2003). Inmersos en la cibercultura (Lévy, 2007), la generación «millennial», "Generación del Milenio» o «Generación Y» (como evolución terminológica de la denominada «Generación X» que les precede) ha introducido además nuevos hitos en el ámbito de la investigación en comunicación (Francese, 2003). La figura de los consumidores activos o «prosumidores» ha adquirido una importancia creciente en los procesos comunicativos, facilitando la posibilidad de convertir a cualquier usuario en un potencial emisor de contenidos de alcance global. La generación «millennial», cuyo rango de edad comprende los nacidos entre 1980 y 1994 (McCrindle y Wolfinger, 2010), consolida un tipo de cibernautas que posee una gran importancia en el ecosistema actual de medios, especialmente en los digitales. Este perfil de usuarios demanda transformaciones que afectan profundamente al escenario educativo. Autores como Piscitelli, Adaime y Binden (2010) han incidido en la necesidad de reformular los procesos formati- 
vos (desde la escuela hasta la universidad) para adaptarlos a los nuevos perfiles de estudiantes y a los cambios tecnológicos, especialmente los vinculados con las redes sociales (en adelante RRSS).

Las investigaciones sobre la utilización de las redes sociales por parte de los jóvenes se han centrado básicamente en las motivaciones de uso, las plataformas más valoradas y, en algunos casos, las tendencias que se pueden desprender a partir de la utilización que hacen de las mismas. Además, la mayoría de estudios se han basado en dos grandes públicos: «jóvenes» en el sentido amplio del término o «universitarios». Este artículo resulta de una investigación que ha sumado a estos dos perfiles la especificidad de una disciplina: la comunicación. Así, se ha focalizado en una muestra de jóvenes universitarios que cursan estudios de comunicación (primer o segundo año) en 16 países iberoamericanos. El principal objetivo es diagnosticar el tipo de valoración y de uso que el alumnado de comunicación - futuros profesionales de los medios- hace de las RRSS.

Las RRSS se consolidan como plataformas donde «todos los miembros de la comunidad saben, conocen y actúan en forma previsible y pertinente» (Galindo, 2010a: 3). Jones et al. (2010) analizaron los tipos de usos que el alumnado realizaba de las plataformas colaborativas. Con una muestra de estudiantes de cinco universidades inglesas, los investigadores cuestionaron que la denominación de «nativos digitales» abarque a usuarios con la misma concepción, las mismas habilidades instrumentales y, especialmente, la misma competencia crítica que sus compañeros en el manejo de las plataformas digitales. Este aspecto introduce un importante desafío para el sistema educativo (Singh et al., 2015), especialmente para las facultades que trabajan en la formación de los futuros profesionales de la Comunicación, ya que una serie de demandas y transformaciones afectan tanto al profesorado como a los estudiantes (Roblyer et al., 2010). En este sentido, un estudio comparativo entre Brasil y Portugal planteaba la necesidad, en ambos países, de una actualización y de un reciclaje formativo de los docentes en las competencias digitales (Silva et al., 2014).

Partiendo de lo anterior, el artículo responde a la siguiente pregunta de investigación de ámbito descriptivo: "¿Qué tipo de uso y qué valoración poseen los estudiantes de Comunicación de Iberoamérica de las redes sociales?». Con relación a ella, se han considerado las siguientes hipótesis de trabajo:

- H1: los estudiantes de Comunicación de Iberoamérica presentan una concepción común de las redes sociales en cuanto a su importancia y su utilidad, más allá de las diferencias socioeconómicas que existen entre estos países.

- H2: el alumnado de Comunicación confiere una gran importancia informativa a las redes sociales por encima de los medios de comunicación.

- H3: existe una confusión terminológica respecto a la definición de red social que poseen los estudiantes de Comunicación. 


\section{Marco teórico}

El estudio de los cambios de la comunicación exige una búsqueda detallada de los orígenes y los porqués de cada proceso (Galindo, 2011), especialmente en el escenario actual marcado por las vertiginosas mediaciones sociales (Martin Serrano, 2008). La instrumentalización de la comunicación, a través de la emergencia de numerosas plataformas y herramientas digitales, ha contribuido a la creación de una ideología técnica (Wolton, 2000). La sociedad digital ha generado importantes transformaciones tecnológicas que han afectado a las dimensiones sociales, laborales, educativas (Piscitelli, 2002). En este contexto, presidido por la web $2.0 \mathrm{o}$ web social, se ha producido la irrupción de nuevos conceptos como prosumidores (Toffler, 1980), inteligencia colectiva (Lévy, 1999) o cultura participativa (Jenkins, 2008) que definen el sistema mediático actual. Asistimos a la consolidación de un nuevo contexto conocido como media ecology, que establece que las tecnologías de la comunicación generan ambientes que afectan a los sujetos que las utilizan. El término ecologia, como apuntaba Postman (1970), impacta en la estructura, el contenido y las personas. Esta ecología defiende que las tecnologías de la comunicación crean ambientes que tienen efectos sobre los sujetos (usuarios) que las utilizan (Scolari, 2012, 2013).

En esta geografía de internet (Castells, 2003b), que ha pasado del multimedia al transmedia, la falacia de la caja negra de Jenkins (2008) adquiere una vigencia renovada y se refuerza el planteamiento de este autor que interpreta la convergencia como un proceso sistémico que aglutina aspectos técnicos, culturales, industriales y sociales. Se trata de una dinámica equiparable a un montaje de sucesivas transformaciones que abarca diferentes ámbitos de la sociedad (Becerra, 2000). Existe, por tanto, una dependencia de los nuevos medios respecto de las convenciones de los viejos (Manovich, 2005). Para Martín-Barbero (2015), el paradigma digital se vincula directamente con modelos anteriores que encuentran su base en la conversación oral y gestual. Esta tendencia a la hibridación es una constante en el proceso de desarrollo y evolución del sistema mediático que demanda de la utilización combinada de las disciplinas que las analizan por separado (García Canclini, 1990). Por tanto, los procesos dialógicos se tornan decisivos. «El conversar es la matriz de lo que hoy se configura en una red social, a la que se entra y de la que se sale entrelazando palabras con fotos, con retazos de música y trazos de dibujos» (Martín-Barbero, 2015: 15).

El contexto latinoamericano se ha caracterizado por presentar en los últimos ańos un álgido crecimiento en el uso de las plataformas on-line, hasta llegar a situarse como una de las regiones del mundo con una mayor utilización de las mismas. Según CEPAL, en 2013 cerca del 78,4\% de los usuarios latinoamericanos tenían presencia en redes sociales. Este porcentaje superaba los resultados obtenidos en escenarios con niveles de acceso y conectividad a internet muy superiores, como América del Norte (64,6\%) o Europa $(54,5 \%)$. De este modo, se detecta que el grado de presencia y utilización de 
las plataformas dialógicas que introduce la Red no está directamente vinculado con la coyuntura socioeconómica de los países. Asimismo, destaca el dato de que Brasil, México, Argentina, Perú, Chile y Colombia se sitúen entre los diez países del mundo con mayor porcentaje de usuarios en el ciberespacio (CEPAL, 2015). Estudios como el de Espinar-Ruiz y González-Río (2015) resaltan la relación entre el uso de internet y una mayor implicación política de los jóvenes. Así, resulta de interés investigar sobre la importancia y utilidad que los estudiantes de este ámbito - estrechamente vinculados con las RRSS y las plataformas tecnológicas en general- confieren a las redes. El artículo analiza y describe "cómo», "con quién» y "para qué» los alumnos de Comunicación utilizan las RRSS.

\section{Metodología}

Los datos de este estudio derivan de un proyecto de investigación iniciado en el curso 2013-2014 y en el que han participado universidades de 16 países: Argentina, Brasil, Chile, Colombia, Costa Rica, Cuba, Ecuador, El Salvador, España, Guatemala, Honduras, México, Nicaragua, Paraguay, Perú y República Dominicana. La investigación reunió un total de 32.400 evidencias, a partir de la participación de 1.080 estudiantes (64\% mujeres y $34 \%$ hombres, del primer o segundo curso académico de Comunicación). Las encuestas, realizadas a través de un formulario on-line integrado por 30 preguntas, se aplicaron entre abril y junio de 2016, con una participación promedio de 68 estudiantes por país. De este modo, se ha llevado a cabo un análisis diagnóstico sobre cómo los estudiantes de Comunicación iberoamericanos conciben, valoran y utilizan las RRSS en sus relaciones sociales y en los procesos de acceso, consulta y producción de contenidos digitales on-line.

En el marco del estudio, la muestra invitada, concebida como el conjunto de elementos de la población a los que se pide que participen en la investigación (Del Rincón et al., 1995), corresponde a un grupo de centros universitarios que imparten estudios de Comunicación en el ámbito iberoamericano, y que han sido seleccionados a partir de rankings y directorios académicos más relevantes ${ }^{1}$.

1. Como THE WUR 2016-2017, QS World University o el directorio elaborado por Quacquarelli Symonds. Instituciones participantes: Universitat Autònoma de Barcelona (España); Universidad Pontificia Bolivariana de Medellín (Colombia); Universidad Nacional Autónoma de Honduras de Tegucigalpa y de San Pedro Sula (Honduras); Universidad Centroamericana de Managua (Nicaragua); Universidad Mariano Gálvez de Ciudad de Guatemala (Guatemala); Universidad Católica del Norte de Antofagasta (Chile); Universidad Autónoma de Nuevo León (México); Universidad de Lima (Perú); Universidad Austral de Buenos Aires (Argentina); Universidad Simeón Cañas y Universidad Gerardo Barrios (El Salvador); Pontificia Universidad Católica Madre y Maestra de Santo Domingo (República Dominicana), Universidad de La Habana (Cuba); Universidad Autónoma de Asunción (Paraguay); Universidad San Francisco de Quito (Ecuador); Universidad Nacional (Costa Rica); Universidade Federal de Alagoas, Universidade de Santa Cruz do Sul y Faculdade Cásper Líbero (Brasil). 
La investigación adoptó una metodología híbrida basada en una encuesta matriz que cruzaba preguntas de tipo cuantitativo con cuestiones abiertas que han permitido un trabajo más cualitativo en la lectura e interpretación de las respuestas (Holsti, 1969), aplicando de este modo las metodologías más adecuadas para este tipo de estudios (Gómez, 1990). Los datos cuantitativos se han analizado a partir de frecuencias y de porcentajes. La encuesta se ha basado en un muestreo aleatorio simple sin reposición.

Las variables principales de las preguntas de los cuestionarios han sido: a) Concepción de una red social (fortalezas y debilidades); b) Importancia de una red social en su vida social y amistades; $c$ ) Reflexión sobre los hábitos de uso de las redes sociales; d) Exposición de las posibles sinergias entre las RRSS y los medios de comunicación; e) Tipo de usuarios con los que se relacionan en sus redes. A través de estos cuestionarios cerrados se ha analizado el nivel de conocimiento, el tipo de uso y la importancia que los estudiantes de comunicación otorgan a las plataformas digitales, especialmente las RRSS en los procesos de detección, selección, consulta y producción de contenidos informativos multimedia.

\section{Resultados}

Galindo (2010b) identifica las RRSS, y en especial Facebook, como plataformas que promueven una cultura basada en la accesibilidad, la diversión y lo sencillo. Así, el estudio interroga a 1.080 estudiantes de Comunicación sobre cómo, con quién y para qué utilizan estas plataformas. El estudio permite, en un primer momento, corroborar la importancia que las RRSS poseen entre el público juvenil. Se identifica que prácticamente la totalidad de los universitarios encuestados $(99,35 \%)$ disponen de un perfil personal en alguna red social. Salvo cinco países (Brasil con un 98,7\%; Colombia, 99,3\%; España, 99,6\%; México, 96,9\%, y Honduras, 98,3\%), en el resto de casos los resultados alcanzan el $100 \%$ del total. De este modo, la investigación parte de un dato irrefutable, que refuerza las conclusiones de las investigaciones anteriormente mencionadas. Los jóvenes estudiantes de Comunicación del escenario iberoamericano están presentes en las RRSS. Además, una holgada mayoría de ellos (79,26\%) afirma que las RRSS no constituyen una moda pasajera, mientras un $17,22 \%$ sí considera que se trata de un fenómeno comunicativo efímero. Llama la atención que, en toda la muestra, sin importar el nivel de penetración de internet o de las diferencias socioeconómicas, casi la totalidad de los estudiantes de Comunicación encuestados encuentran en las RRSS un espacio en el que necesariamente deben estar.

Partiendo de estos datos generales, el análisis diagnóstico detecta un claro predominio de Facebook y Twitter (Tabla 1). La primera de ellas se sitúa, en todos los casos, en cifras cercanas o superiores al $90 \%$. España, paradójicamente, es el país donde la presencia en esta red es más reducida $(89,19 \%)$, aunque la participación es muy elevada. Las respuestas de los estudiantes, que podían señalar más de una opción, refuerzan la tendencia mundial de creci- 
Tabla 1. Universitarios de Comunicación y RRSS

\begin{tabular}{lcccc}
\hline \multicolumn{5}{c}{ ¿En qué redes sociales están los estudiantes de comunicación de lberoamérica? } \\
\hline & ARGENTINA & BRASIL & CHILE & COLOMBIA \\
\hline Facebook & $98,28 \%$ & $100 \%$ & $98,51 \%$ & $97,10 \%$ \\
Twitter & $87,93 \%$ & $58,97 \%$ & $59,70 \%$ & $73,91 \%$ \\
Linkedln & $12,07 \%$ & $28,21 \%$ & $2,99 \%$ & $15,22 \%$ \\
Instagram & $55,17 \%$ & $48,72 \%$ & $47,76 \%$ & $0,00 \%$ \\
Snapchat & $12,07 \%$ & $34,62 \%$ & $10,45 \%$ & $0,00 \%$ \\
\hline & COSTA RICA & CUBA & ECUADOR & EL SALVADOR \\
\hline Facebook & $100 \%$ & $100 \%$ & $100 \%$ & $100 \%$ \\
Twitter & $80 \%$ & $62,50 \%$ & $100 \%$ & $42,86 \%$ \\
Linkedln & $20 \%$ & $41,67 \%$ & $14,29 \%$ & $4,76 \%$ \\
Instagram & $60 \%$ & $4,17 \%$ & $28,57 \%$ & $28,57 \%$ \\
Snapchat & $40 \%$ & $0,00 \%$ & $0,00 \%$ & $4,76 \%$ \\
\hline & ESPAÑA & GUATEMALA & HONDURAS & MÉXICO \\
\hline Facebook & $89,19 \%$ & $95,77 \%$ & $97,46 \%$ & $95,38 \%$ \\
Twitter & $80,18 \%$ & $78,87 \%$ & $37,29 \%$ & $66,15 \%$ \\
Linkedln & $27,93 \%$ & $12,68 \%$ & $5,08 \%$ & $9,23 \%$ \\
Instagram & $41,44 \%$ & $33,80 \%$ & $17,80 \%$ & $0,00 \%$ \\
Snapchat & $5,41 \%$ & $15,49 \%$ & $7,63 \%$ & $0,00 \%$ \\
\hline & NICARAGUA & PARAGUAY & PERÚ & R. DOMINICANA \\
\hline Facebook & $100 \%$ & $95,24 \%$ & $100 \%$ & $90,32 \%$ \\
Twitter & $79,45 \%$ & $85,71 \%$ & $63,33 \%$ & $74,19 \%$ \\
Linkedln & $19,18 \%$ & $14,29 \%$ & $23,33 \%$ & $19,35 \%$ \\
Instagram & $39,73 \%$ & $33,33 \%$ & $28,33 \%$ & $64,52 \%$ \\
Snapchat & $13,70 \%$ & $14,29 \%$ & $11,67 \%$ & $9,68 \%$ \\
\hline
\end{tabular}

Fuente: elaboración propia.

miento de las dos plataformas en el escenario iberoamericano que otros estudios también han identificado, y que sitúan a Facebook como la principal red en uso y en perspectivas de crecimiento futuro. Según datos del Instituto para la Integración de América Latina y la Corporación Latinobarómetro (INTAL y Latinobarómetro, 2016), el 57\% de los usuarios tenía presencia en Facebook, WhatsApp o YouTube, a pesar de haber tenido dificultades para alimentarse, y el $51 \%$ no tenía acceso a agua potable. Además, el $81 \%$ de los menores de 24 ańos estaban conectados a Facebook frente a un $10 \%$ de mayores de 65. En la misma línea, el estudio de CEPAL (2015) reforzaba la tendencia e indicaba que Facebook era la red social líder en el contexto latinoamericano, con 145 millones de visitantes únicos.

Los datos confirman la tendencia de crecimiento y refuerzan el liderazgo de Facebook para el perfil de usuario analizado. Vale la pena decir, en cualquier caso, que los resultados de Facebook en España pueden indicar que la red social más grande del mundo puede disputarse el liderazgo en el público juvenil en los próximos años y en algunos países específicos. Por su parte, 
Twitter se sitúa como la segunda red social más utilizada. Salvo en los casos de El Salvador $(42,86 \%)$ y Honduras $(37,29 \%)$, el porcentaje de presencia en esta plataforma de microbloging en el resto de países supera el $50 \%$ del total.

Las principales diferencias entre los escenarios estudiados vienen dadas, no obstante, con las demás plataformas seleccionadas por los alumnos. En estos casos, no es posible identificar una valoración y tipo de uso similar en el conjunto de países. LinkedIn, por ejemplo, centrada en la oferta y búsqueda de oportunidades laborales, ofrece resultados muy dispares. Aunque existe una propensión de uso mayoritaria que le sitúa en las preferencias de $20 \%$ del alumnado, no es una preferencia uniforme. La variedad de porcentajes es muy amplia y se dan casos muy significativos como el de Cuba que, con el $41,67 \%$, se coloca como el país donde los estudiantes muestran un mayor uso (o preferencia de uso) de LinkedIn; mientras que Chile (con un 2,99\%) es el que posee un porcentaje menor.

Finalmente, los casos de Instagram y Snapchat resultan llamativos porque, en diversos países, se detecta un uso nulo de ambas, pese a que son plataformas de gran calado entre los jóvenes. Por ejemplo, en México y Colombia los estudiantes dicen no utilizar estas plataformas de publicación de contenidos fotográficos (en ambos casos, el resultado global es de $0 \%$ ). Estos datos sorprenden, ya que estudios recientes identificaban un crecimiento de Instagram en América Latina. Según Latamclick (2017), Latinoamérica tiene más de 60 millones de usuarios registrados en Instagram (28 millones de hombres; 32 millones de mujeres aproximadamente). Además, los datos sobre México y Colombia resultan opuestos a los resultados de nuestra investigación. México aparece como el país latinoamericano con mayor presencia en Instagram, con 16 millones de usuarios (7,5 millones de hombres; 8,6 millones de mujeres). Asimismo, Colombia ocupa el tercer lugar en usuarios de esta plataforma, con 9.200 .000 registros. Aunque nuestra investigación se centra únicamente en un perfil concreto de usuarios (estudiantes de Comunicación de primer y segundo año de carrera), los registros de estos países advierten de alguna particularidad que debe estar relacionada con aspectos sociales, terminológicos o de concepción de las características y el alcance de las plataformas.

Sobre Snapchat, los resultados confrontan con datos de la consultoría eMarketer, que apuntaban que el $23 \%$ de la población en Latinoamérica utiliza apps de mensajería, y entre las plataformas más usadas se encuentran WhatsApp, Facebook Messenger, Snapchat y WeChat (Tecnósfera, 2015). La segunda edición del IMS Mobile in LatAm Study (ComScore y IMS, 2016), informe sobre consumo digital y aplicaciones móviles en Brasil, México, Argentina, Colombia, Perú y Chile, señalaba que las aplicaciones de video, social media y mensajería fueron las más populares entre los consumidores. Concretamente, la investigación situaba entre las más utilizadas a WhatsApp, YouTube, Facebook, Facebook Messenger, Google Maps, Instagram y Twitter (que encabezaba la lista). Además, el estudio indicaba que las plataformas Snapchat y Spotify poseen una gran receptividad entre los millennials. En España, el balance es igualmente contradictorio. Se trata del país de 
Europa con mayor presencia en WhatsApp (Comisión Europea, 2016). Además, el 89,8\% de los usuarios de telefonía móvil poseen esta aplicación. De ellos, casi la mitad (42,3\%) afirma que la usa "continuamente» y el 47,5\%, "varias veces al día». Las plataformas dialógicas que dominan entre los españoles son WhatsApp (92,8\%), Facebook (87\%), Twitter (48,9\%) e Instagram $(40,4 \%)$, según la Asociación para la Investigación de Medios de Comunicación (AIMC, 2017).

Esta diferencia en los resultados de preferencia de Instagram y Snapchat entre nuestro trabajo y otras investigaciones podría derivarse de una confusión terminológica entre el alumnado de Comunicación. Esto es: los estudiantes no conciben dentro de la categoría de «red social» a plataformas como Instagram o Snapchat y únicamente sitúan dentro de esa denominación a Facebook y Twitter, principalmente. Como se verá más adelante, a pesar de que el uso es homogéneo en todos los países de la muestra, el perfil de descripción o de definición de las características de una red social varía mucho entre los encuestados sin que exista uniformidad en las respuestas. Los resultados divergentes pueden deberse también a que los estudiantes no quieran reconocer dentro del ámbito académico su pertenencia a este tipo de redes por prejuicios o estereotipos vinculados con las mismas.

Por otro lado, la elaboración de un diagnóstico del perfil tipo de usuario de RRSS en los estudiantes de Comunicación iberoamericanos nos llevó a preguntarles por la edad en que comenzaron a utilizar estas plataformas (Figura 1). Se observa que un $87,6 \%$ se inició entre los 10 y los 17 años. De ellos, un $43 \%$ empezó a utilizar RRSS antes de cumplir los 14 años. Para todos los países de la muestra se detecta un problema, relativo a la iniciación

Figura 1. Iniciación en RRSS

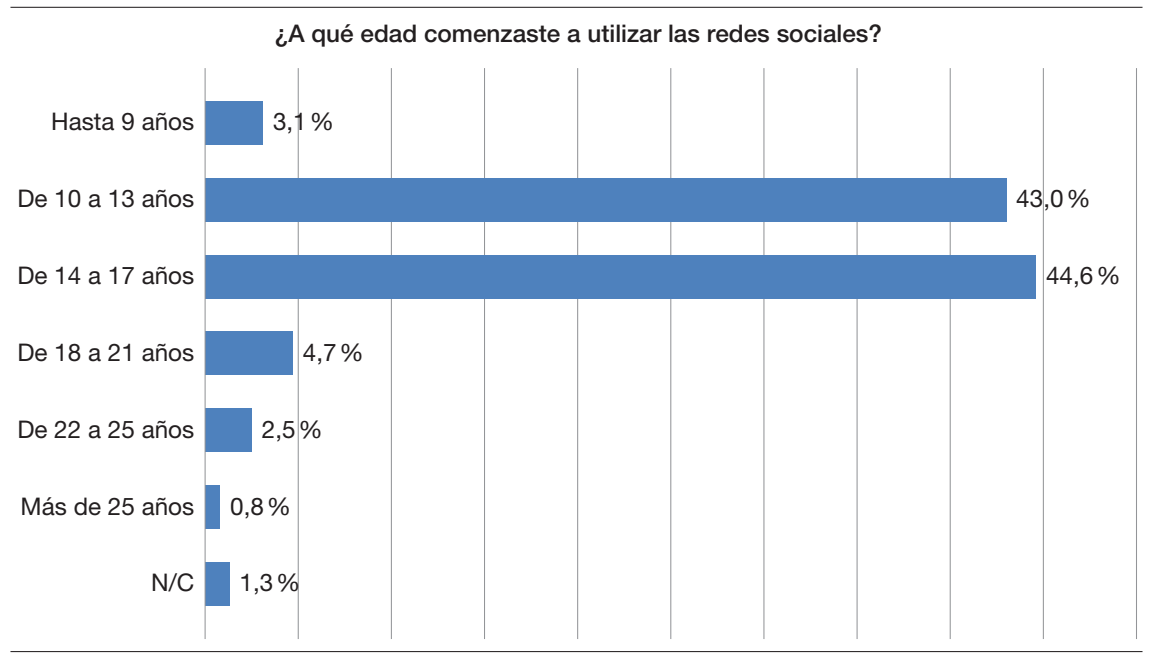

Fuente: elaboración propia. 
precoz y, por ende, vinculado al uso seguro de internet. Este último dato alerta de la necesidad de impulsar acciones de sensibilización y alfabetización digital mediática que preparen a los usuarios — de edades tan tempranaspara el uso crítico, responsable y ético de dichas plataformas.

Estos resultados recomiendan el desarrollo de investigaciones sobre el llamado «internet sano» (García, 2012) y sobre la alfabetización digital mediática (Pérez Tornero y Tejedor, 2016) de los internautas, concebida como el proceso de utilizar herramientas de tecnología, comunicación digital o redes para acceder, manejar, integrar, crear, evaluar información para poder funcionar con solvencia en una sociedad del conocimiento (Pérez Tornero, 2016).

Las principales RRSS establecen una edad mínima para acceder a las mismas. Con 13 años se puede estar presente en Twitter, Tumblr, Pinterest, WhatsApp y Foursquare, entre otras. En caso de permiso paterno, a partir de los 13 años es posible crear perfiles en YouTube, Wechat o Flickr. Ya con 14 años, los usuarios pueden registrarse en LinkedIn, Instagram o Facebook. En el caso de Snapchat, se alude a una franja de edad de entre 13 y 17 años (San José, 2017).

A pesar de estas limitaciones, la investigación permite identificar que la mayoría de los alumnos de Comunicación comienza a crear perfiles y a utilizar las RRSS de forma prematura en Iberoamérica. Junto a esta temprana iniciación, la importancia que el alumnado confiere a estas plataformas está vinculada al protagonismo que les dan en el marco de sus relaciones sociales. En este sentido, un 63,15\% asegura que «todos» sus amigos están agregados a su perfil de red social; mientras que solo un 31,48\% afirma tener amistades más allá del directorio de contactos de su perfil digital. A ello se une la aseveración (Figura 2) que indica que para una mayoría de los estudiantes $(66,20 \%)$, su vida social «ha cambiado con el uso de las RRSS».

Figura 2. Vida social y RRSS

¿Ha cambiado tu vida social con el uso de las redes sociales?

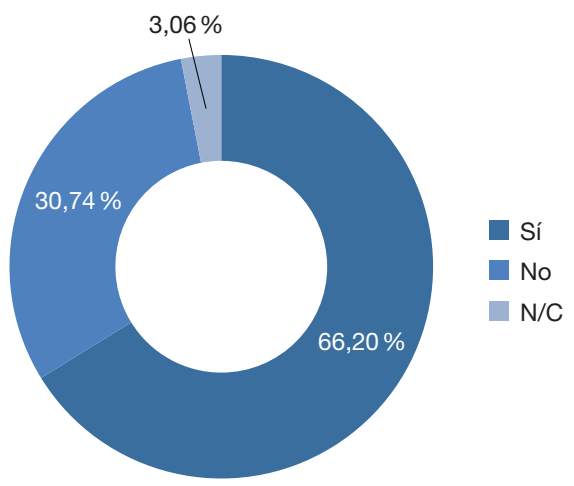

Fuente: elaboración propia. 
Salvo Paraguay, en todos los países analizados la mayoría de los estudiantes de Comunicación afirma que las RRSS «han cambiado su vida social» (Tabla 2), resultados que se pueden vincular con estudios que sitúan a Costa Rica $(78 \%)$ y Chile $(69 \%)$ como dos de los países con mayor penetración de las redes sociales en el contexto latinoamericano (INTAL y Latinobarómetro, 2016). Los resultados denotan la consolidación de un escenario comunicativo (Castells, 2003a) asociado a la segunda década del siglo XXI y que delinea transformaciones importantes en diferentes ámbitos de la sociedad, como la socialización, el consumo de información y la construcción de estatus social (Giraldo-Luque et al., 2017) en los jóvenes universitarios inmersos de lleno en la cibercultura (Lévy, 2007).

Tabla 2. Vida social y RRSS por países

\begin{tabular}{|c|c|c|c|c|}
\hline \multicolumn{5}{|c|}{ ¿Ha cambiado tu vida social con el uso de las redes sociales? } \\
\hline & ARGENTINA & BRASIL & CHILE & COLOMBIA \\
\hline Sí & $65,52 \%$ & $73,08 \%$ & $80,60 \%$ & $62,32 \%$ \\
\hline No & $29,31 \%$ & $23,08 \%$ & $17,91 \%$ & $34,78 \%$ \\
\hline \multirow[t]{2}{*}{$\mathrm{N} / \mathrm{C}$} & $5,17 \%$ & $3,85 \%$ & $1,49 \%$ & $2,90 \%$ \\
\hline & COSTA RICA & CUBA & ECUADOR & EL SALVADOR \\
\hline Sí & $80 \%$ & $54,17 \%$ & $85,71 \%$ & $64,29 \%$ \\
\hline No & $20 \%$ & $41,67 \%$ & $0,00 \%$ & $26,19 \%$ \\
\hline \multirow[t]{2}{*}{$\mathrm{N} / \mathrm{C}$} & $0,00 \%$ & $4,17 \%$ & $14,29 \%$ & $9,52 \%$ \\
\hline & ESPAÑA & GUATEMALA & HONDURAS & MÉXICO \\
\hline Sí & $71,17 \%$ & $67,61 \%$ & $50,85 \%$ & $76,92 \%$ \\
\hline No & $27,93 \%$ & $32,39 \%$ & $44,92 \%$ & $18,46 \%$ \\
\hline \multirow[t]{2}{*}{$\mathrm{N} / \mathrm{C}$} & $00,90 \%$ & $0,00 \%$ & $4,24 \%$ & $4,62 \%$ \\
\hline & NICARAGUA & PARAGUAY & PERÚ & R. DOMINICANA \\
\hline Sí & $53,42 \%$ & $38,10 \%$ & $78,33 \%$ & $64,52 \%$ \\
\hline No & $41,10 \%$ & $61,90 \%$ & $18,33 \%$ & $35,48 \%$ \\
\hline $\mathrm{N} / \mathrm{C}$ & $5,48 \%$ & $0,00 \%$ & $3,33 \%$ & $0,00 \%$ \\
\hline
\end{tabular}

Fuente: elaboración propia.

Esta investigación también indagó en el tipo de relaciones que los universitarios establecen a través de sus RRSS, es decir, con qué tipo de usuarios se relacionaban virtualmente (Figura 3). El alumnado, que podía contestar más de una opción, señaló mayoritariamente que sus interacciones en línea se dan con "amigos». Así, se refuerza el componente de la «amistad», que además está vinculado con el hecho que una mayoría de los estudiantes afirma que «todos» sus amigos están agregados a su perfil (o perfiles) en RRSS. Los resultados de las interacciones de los jóvenes en las RRSS fortalecen así la idea de la burbuja mediática sugerida por Eli Pariser (2011). El refuerzo que realizan las RRSS de los lazos ya construidos en el entorno off line limita también el rango de divergencia, pluralidad y alcance al que pueden acceder los usuarios con el potencial de las mismas redes (Brundidge, 2010). 
Figura 3. Interacciones en las RRSS

¿Con qué tipo de personas te relacionas cuando te conectas?

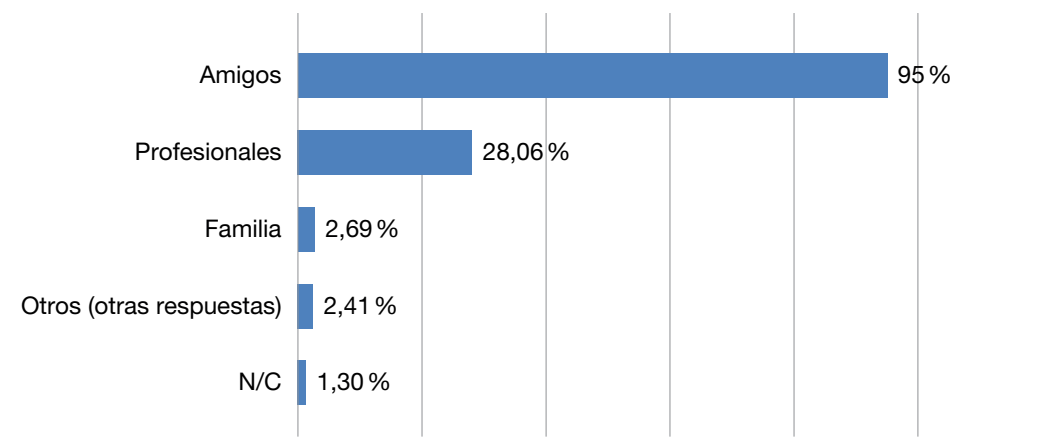

Fuente: elaboración propia.

En su enfoque diagnóstico, el estudio ha permitido identificar que en los 16 países analizados (Tabla 3), los estudiantes coinciden en señalar que el tipo de usuarios con los que se relacionan cuando se conectan a sus perfiles de redes sociales son «amigos». La vinculación entre el uso de RRSS y las relaciones de amistad quedan verificadas. El porcentaje de alumnos que escogieron la opción de «amigos» se sitúa en todos los países (salvo Costa Rica) por encima del $85 \%$ del total. En segundo lugar, se sitúa el tipo de intercambios con usuarios que identifican dentro de un escenario "profesional». De este modo, se puede señalar que la combinación, dentro de la misma plataforma, de las relaciones «sociales» con las "profesionales» es un aspecto muy consolidado en el uso que los jóvenes estudiantes de Comunicación realizan en sus RRSS. La amistad y el trabajo conviven en estas plataformas por encima de los intercambios con integrantes del seno familiar o perfiles de otra tipología.

La importancia que los estudiantes de Comunicación confieren a las RRSS queda plasmada en otra elección (Figura 4). Alrededor del $60 \%$ de los universitarios encuestados indican su preferencia por una red social frente a páginas web de medios digitales. Este aspecto resulta especialmente interesante, ya que se trata de futuros profesionales de la Comunicación, lo que nos permite pensar que el alumnado valoraría positivamente la posibilidad de trabajar en internet (o, concretamente, en una red social).

Se observa, como apuntaban Bulut y Doğan (2017), una concepción de las redes como posibles escenarios laborales. Este aspecto contribuye a subrayar la necesidad de cambios en los planes curriculares de los estudios de comunicación para adaptar los perfiles profesionales de los futuros egresados a las demandas del mercado (Salaverría, 2016; Roblyer et al., 2010). Para lograrlo, una de las principales metas sería la actualización de los programas académicos, la reformulación de los enfoques en los estudios y la inserción de estrategias para el reciclaje continuado del profesorado (Tramullas, 2016). 
Tabla 3. Interacciones en las RRSS por países

\begin{tabular}{|c|c|c|c|c|}
\hline \multicolumn{5}{|c|}{ ¿Con qué tipo de personas te relacionas cuando te conectas? } \\
\hline & ARGENTINA & BRASIL & CHILE & COLOMBIA \\
\hline Amigos & $96,55 \%$ & $98,72 \%$ & $98,51 \%$ & $97,10 \%$ \\
\hline Profesionales & $37,93 \%$ & $35,90 \%$ & $16,42 \%$ & $25,36 \%$ \\
\hline Familia & $3,45 \%$ & $8,97 \%$ & $2,99 \%$ & $0,72 \%$ \\
\hline Otras & $3,45 \%$ & $3,85 \%$ & $0,00 \%$ & $0,72 \%$ \\
\hline \multirow[t]{2}{*}{$\mathrm{N} / \mathrm{C}$} & $1,72 \%$ & $1,28 \%$ & $0,00 \%$ & $0,72 \%$ \\
\hline & COSTA RICA & CUBA & ECUADOR & EL SALVADOR \\
\hline Amigos & $80 \%$ & $100 \%$ & $100 \%$ & $95,24 \%$ \\
\hline Profesionales & $40 \%$ & $45,83 \%$ & $28,57 \%$ & $28,57 \%$ \\
\hline Familia & $0,00 \%$ & $8,33 \%$ & $0,00 \%$ & $0,00 \%$ \\
\hline Otras & $0,00 \%$ & $12,50 \%$ & $0,00 \%$ & $0,00 \%$ \\
\hline \multirow[t]{2}{*}{$\mathrm{N} / \mathrm{C}$} & $0,00 \%$ & $0,00 \%$ & $0,00 \%$ & $4,76 \%$ \\
\hline & ESPAÑA & GUATEMALA & HONDURAS & MÉXICO \\
\hline Amigos & $95,50 \%$ & $88,73 \%$ & $89,83 \%$ & $96,92 \%$ \\
\hline Profesionales & $27,93 \%$ & $28,17 \%$ & $24,58 \%$ & $13,85 \%$ \\
\hline Familia & $0,45 \%$ & $2,82 \%$ & $5,08 \%$ & $1,54 \%$ \\
\hline Otras & $0,90 \%$ & $5,63 \%$ & $3,39 \%$ & $1,54 \%$ \\
\hline \multirow[t]{2}{*}{$\mathrm{N} / \mathrm{C}$} & $1,80 \%$ & $1,41 \%$ & $1,69 \%$ & $1,54 \%$ \\
\hline & NICARAGUA & PARAGUAY & PERÚ & R. DOMINICANA \\
\hline Amigos & $95,89 \%$ & $85,71 \%$ & $96,67 \%$ & $90,32 \%$ \\
\hline Profesionales & $46,58 \%$ & $52,38 \%$ & $13,33 \%$ & $22,58 \%$ \\
\hline Familia & $5,48 \%$ & $4,76 \%$ & $0,00 \%$ & $0,00 \%$ \\
\hline Otras & $2,74 \%$ & $4,76 \%$ & $3,33 \%$ & $3,23 \%$ \\
\hline $\mathrm{N} / \mathrm{C}$ & $0,00 \%$ & $0,00 \%$ & $0,00 \%$ & $3,23 \%$ \\
\hline
\end{tabular}

Fuente: elaboración propia.

La preferencia universitaria por las RRSS frente a las cabeceras digitales adquiere una dimensión mayor cuando se pregunta a los estudiantes por la relación entre medios de comunicación on-line y redes sociales. La mayoría otorga un mayor protagonismo a las RRSS dentro de sus plataformas informativas (Figura 5). La prevalencia de las RRSS sobre cualquier otro dispositivo de comunicación —que comprende no solo el ámbito informativo, del cual adquiere la primera posición, sino que también define el marco de interacción social parcialmente limitado al universo de amistades establecidopermite vislumbrar la concentración de diferentes funciones sociales en unas únicas plataformas interactivas. El cambio y la concentración son evidentes para todos los países estudiados y definen a las RRSS como escenarios que, si bien pueden variar con el tiempo, tienen la capacidad de resistir al cambio, de adaptarse, e incluso de propiciarlo a medida que incluyen más actividades y funciones sociales dentro de sus propios entornos mediáticos digitales. En el ámbito de la comunicación, los resultados del estudio son contundentes y definen tanto un alto interés en los nuevos medios asociados a las RRSS, 
Figura 4. Preferencias de uso

¿Prefieres una red social o un medio de comunicación en línea?

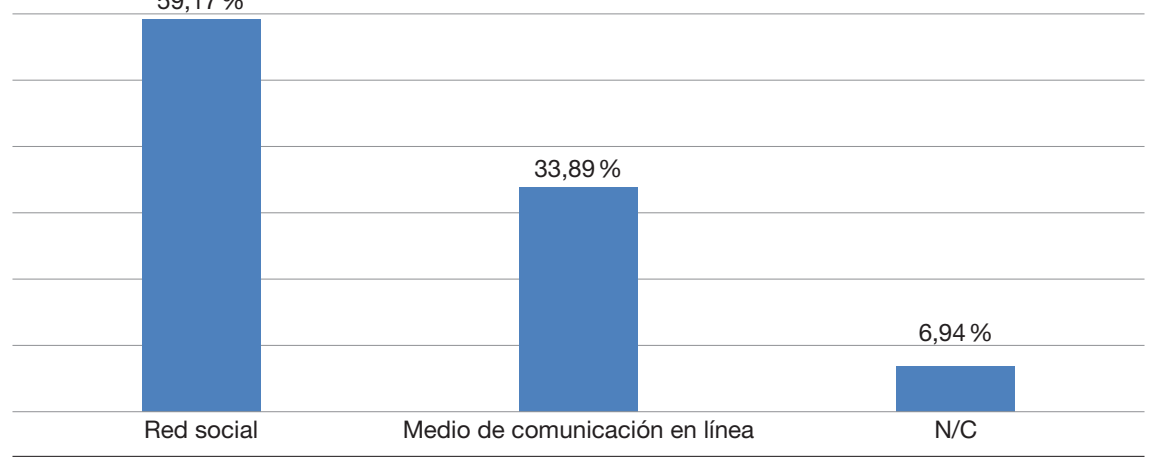

Fuente: elaboración propia.

como una posición dominante en el imaginario social de los jóvenes estudiantes de comunicación de las RRSS frente a otros espacios tradicionales de comunicación e incluso de ejercicio profesional.

Finalmente, la investigación advierte de la existencia de una seria confusión terminológica en la noción que parte del alumnado tiene sobre conceptos decisivos del escenario comunicativo. En las preguntas de carácter cualitativo, sus respuestas denotan que desconocen las características que diferencian a un «nativo» de un «inmigrante» digital. Se detecta que los estudiantes consideran que un "nativo» digital posee, en todos los casos, una mayor solvencia que un «inmigrante» digital en el manejo instrumental de las plataformas

Figura 5. Medios on-line y RRSS

¿Crees que los medios de comunicación en línea deberían incorporar una red social en su plataforma?

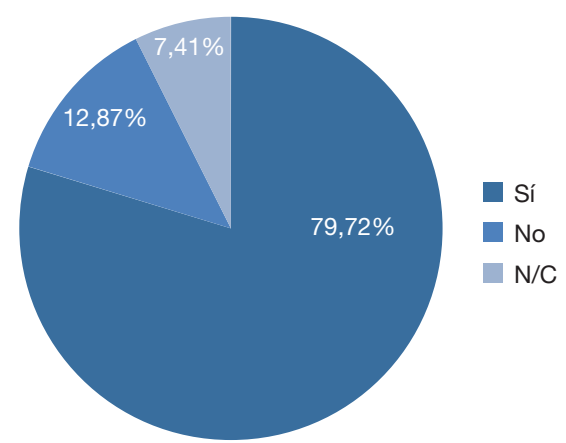

Fuente: elaboración propia. 
Tabla 4. Opinión sobre nativos e inmigrantes digitales

¿Qué diferencias señalarías entre un nativo digital y un inmigrante digital en el uso de una red social?

«El nativo es alguien que consume constantemente. El inmigrante es meramente curioso».

«El nativo generalmente sabe qué cosas publicar y cuáles no. El inmigrante necesita de un tiempo para aprender».

«El nativo digital sabe muy bien cómo usar las redes sociales y sus condiciones de usos y seguridad. Los inmigrantes suelen tenerle un poco de rechazo al hecho de compartir cosas "íntimas" en el internet que puede estar al alcance de todos".

«El nativo digital utiliza las redes más creativamente, sus publicaciones en la web tienen un objetivo claro. El inmigrante digital quizás no comprende el alcance que puede llegar a tener lo que hace o no con su perfil en la web".

«La manera en que se usa varía entre un adulto y un joven. El adulto le da un perfil más serio».

«Un nativo tiene mejor manejo no de una, sino varias redes sociales; no así un inmigrante, el cual está recién conociendo una red social en particular».

«Principalmente a facilidade e a dificuldade de lidar com a rede social e suas ferramentas».

Fuente: elaboración propia.

digitales (Tabla 4). Numerosos alumnos afirman que el primero usa correctamente las RRSS, mientras que el segundo no es capaz. Además, identifican ambos conceptos con los de «joven» y «adulto», respectivamente. Existe, por tanto, la necesidad de clarificar el alcance de este tipo de acepciones e introducir una reflexión que vaya más allá de los aspectos centrados en el uso técnico o instrumental de las plataformas digitales.

Esta particular concepción de los actores del escenario digital por parte del alumnado se observa igualmente cuando se les pregunta por las potencialidades y debilidades de las RRSS. Los alumnos señalan básicamente como elementos positivos de una red social aspectos vinculados con los procesos comunicativos (Figura 6). Los conceptos de «instantaneidad», "comunicación» e «información» son, por este orden, los que definen mejor qué es una red social para los universitarios. A continuación, se sitúan los términos «masivo», «alcance», «libertad», «interactividad», «entretenimiento» $\mathrm{y}$ «socializar». En conclusión, las RRSS son, ante todo, espacios para el intercambio rápido de mensajes que poseen una importancia crucial en los procesos de información y de comunicación. La faceta comunicativa de las plataformas es la que más valoran los estudiantes, que las identifican como herramientas para "socializar», "movilizar» e incluso como instrumentos que potencian la «libertad».

Los elementos negativos más seńalados (Figura 6) aluden a aspectos que influyen negativamente en los procesos comunicativos: "desinformación", «mentiras» $\mathrm{y}$ «rumores». Los estudiantes mencionan además aspectos relacionados con el uso sano de internet («privacidad», «inseguridad» y «adicción»). Existe, por tanto, un conocimiento de riesgos derivados de un mal uso de la red.

En este sentido, Martín-Barbero (2009) incide en el reto de transformar la institución educativa con el objetivo de comprender y aplicar el sentido 
Figura 6. Concepción de una red social

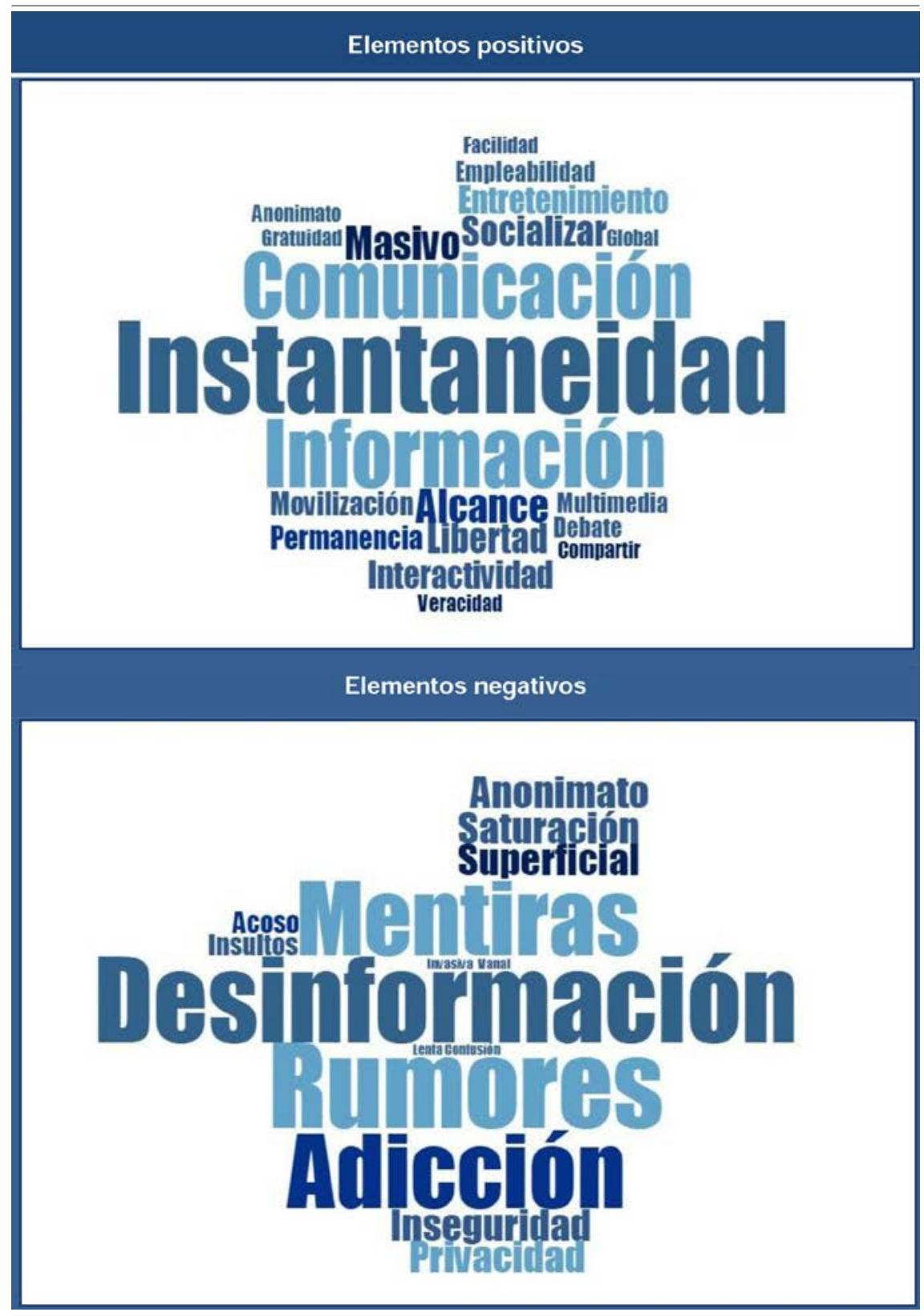

Fuente: elaboración propia. 
global que tiene el verbo navegar, concebido como exploración, manejo y riesgo. El autor alude al potencial alternativo de las mediaciones tecnológicas como una vía de gran provecho en el escenario formativo que pueden contribuir al desarrollo de nuevos procesos y relaciones entre los usuarios y los procesos de lectura y escritura. Ferrés y Piscitelli (2012) también insisten en la necesidad de fomentar una educación mediática que potencie dos aspectos: la producción de mensajes propios y la interacción con mensajes ajenos. Esta propuesta, basada en la combinación de la revolución tecnológica con la neurobiológica, introduce la necesidad de reformular los procesos educativos a partir de los nuevos perfiles de usuarios y de las nuevas plataformas digitales de información y comunicación.

\section{Conclusiones}

El estudio presentado responde al objetivo de desarrollar una aproximación diagnóstica alrededor de una región geográfica con numerosos elementos diferenciadores — entre países - , pero con aspectos que los relacionan e identifican en el uso de las RRSS. Así, el artículo presenta datos que sitúan en un mismo nivel de utilización (en cuanto al uso de las redes sociales por jóvenes universitarios) a países con marcadas diferencias en sus respectivas coyunturas socioeconómicas. El ánimo de diagnóstico descriptivo sobre los usos y motivaciones de los estudiantes de comunicación en América Latina arroja dos oportunidades relevantes. Por una parte, propone resultados que, siendo descriptivos, permiten identificar líneas de trabajo potenciales ligadas a hipótesis más explicativas sobre el uso de las RRSS por parte de la población analizada con la misma intención de hallar similitudes y diferencias entre las intenciones y motivaciones de uso de las RRSS en los jóvenes.

$\mathrm{Al}$ mismo tiempo, los resultados presentan una suerte de mapeo comparativo alrededor de un escenario que ha sido objeto de estudios relativos al uso de las RRSS por jóvenes en un solo país, pero no de forma conjunta y alrededor de un público específico (jóvenes universitarios del ámbito de la comunicación). La perspectiva comparativa permite trazar una visión más amplia de un fenómeno que parece tener y tejer comportamientos homogéneos que superan las diferencias geográficas y que integra, en sí mismo, un objeto de estudio principal para los estudios de comunicación: la incorporación de prácticas y concepciones comunicativas igualitarias en diferentes regiones y países a partir del uso e incorporación de las RRSS en la vida cotidiana de los jóvenes.

La investigación ha permitido, desde su enfoque descriptivo, elaborar un diagnóstico del perfil general de usuario que representan los universitarios del ámbito de la comunicación respecto a las redes sociales. Se ha comprobado (hipótesis 01) que los estudiantes iberoamericanos presentan una concepción común de las RRSS en cuanto a su importancia y utilidad, más allá de las diferencias socioeconómicas que existen entre sus países. Salvo aspectos puntuales, los resultados indican que dichos universitarios conciben, valoran y 
utilizan las redes siguiendo patrones similares. También se concluye que (hipótesis 02) el alumnado de Comunicación confiere una gran importancia informativa a las RRSS por encima de los medios de comunicación. Los futuros comunicadores definen estas plataformas como espacios de gran valor y utilidad para acceder a contenidos informativos e incluso las prefieren frente a las páginas de los cibermedios. Finalmente, el estudio constata (hipótesis 03) que existe una confusión terminológica respecto al concepto de red social entre los estudiantes de Comunicación.

El alumnado asegura que las RRSS han tenido un amplio impacto en su vida social y añaden que no se trata de un fenómeno pasajero, sino que lo consideran como una tendencia cada vez más consolidada. Este alto grado de aceptación de las RRSS entre los usuarios jóvenes y las perspectivas de incremento que se vaticinan en este ámbito ha posibilitado la detección de algunos retos que afectan, especialmente, a la universidad. Es necesario potenciar la presencia de este tipo de contenidos en la formación de los estudiantes de Comunicación para fomentar una mirada crítica hacia las herramientas tecnológicas y un conocimiento más detallado de las oportunidades laborales que ofrece la industria con relación a las RRSS.

La temprana edad de acceso a las redes sociales y la importancia que les confieren en sus relaciones sociales invitan a la reflexión. Más allá de las acciones que se deben adoptar desde los gobiernos y las instituciones, se plantea la necesidad de investigar y generar espacios de análisis y discusión de los aspectos vinculados con el uso sano de internet, las edades de acceso a las RRSS y el tipo de aprovechamiento de estas (los estudiantes decían destinar la mayor parte de su tiempo en estas plataformas al ocio y al entretenimiento).

\section{Referencias bibliográficas}

AsOCIACIÓn PARA la InVESTIGACIÓN DE MEDIOS DE COMUNiCACIÓN (AIMC) (2017). 19० Navegantes en la Red. [Fecha de consulta: 20/07/2017]. <https:// www.aimc.es/a1 mc-c0nt3nt/uploads/2017/05/macro2016.pdf>

BarabÁsI, A-L. y BONabeaU, E. (2003). «Scale-free Networks». Scientific American, 288 (5), 50-59.

$<$ https://doi.org/10.1038/scientificamerican0503-60>

Bauman, Z. (2003). Modernidad líquida. México: Editorial Fondo de Cultura Económica.

BECERRA, M. (2000). «De la divergencia a la convergencia en la sociedad informacional: fortalezas y debilidades de un proceso inconcluso». Zer - Revista de Estudios de Comunicación, 5 (8), 93-112.

BRUNDIDGE, J. (2010). "Encountering "difference" in the contemporary public sphere: The contribution of the Internet to the heterogeneity of political discussion networks». Journal of Communication, 60 (4), 680-700. <https://doi.org/10.1111/j.1460-2466.2010.01509.x>

BuluT, Z. A. y DOĞAN, O. (2017). «The ABCD typology: Profile and motivations of Turkish social network sites users». Computers in Human Behavior, 67 (2), 73-83. $<$ http://dx.doi.org/10.1016/j.chb.2016.10.021> 
CASTElls, M. (2003a). L'Era de la Informació. La societat xarxa. Barcelona: Editorial UOC. Edició especial per a la Generalitat de Catalunya.

- (2003b). La galaxia internet: reflexiones sobre internet, empresa y sociedad. Barcelona: Random House.

COMISIÓN ECONÓMICA PARA LA AMÉRICA LATINA Y EL CARIBE (CEPAL) (2015). La nueva revolución digital. De la internet del consumo a la internet de la producción. <http://repositorio.cepal.org/bitstream/handle/11362/38604/4/S1600780_ es.pdf> [Fecha de consulta: 20/07/2017].

COMISIÓN EUROPEA (2016). Special Eurobarometer 438: E-Communications and the Digital Single Market. <http://ec.europa.eu/information_society/newsroom/ image/document/2016-22/sp438_eb84_2_ecomm_summary_en_15829.pdf> [Fecha de consulta: 10/10/2017].

COMSCORE; IMS (2016). IMS Mobile in LatAm Study, 2a edición, septiembre. <http://insights.imscorporate.com/mobile2016> [Fecha de consulta: 10/10/2017].

Del Rincón, D.; Arnal, J.; LATOrRe, A. y SAns, A. (1995). Técnicas de investigación en ciencias sociales. Madrid: Dykinson.

ESPINAR RUIZ, E. y GONZÁLEZ-RÍO, M. J. (2015). «Uso de internet y prácticas políticas de los jóvenes españoles». Convergencia. Revista de Ciencias Sociales, 69, 13-38. <https://doi.org/10.29101/crcs.v22i69.3632>

FERRÉS, J. y PISCITELLI, A. (2012). «La competencia mediática: propuesta articulada de dimensiones e indicadores». Comunicar, 38, 75-82. $<$ https://doi.org/10.3916/C38-2012-02-08>

FranCESE, P. (2003). "Trend Ticker: Ahead of the Next Wave». AdAge. <http:// adage.com/article/american-demographics/trend-ticker-ahead-wave/44208> [Fecha de consulta: 29/06/2017].

GALINDO, J. (2010a). «La internet y sus redes sociales. Comunicología e ingeniería en Comunicación Social de un fenómeno aun emergente». Razón y Palabra, 71.

- (2010b). «El nuevo nicho civilizatorio del ciberespacio: La red social como un desarrollo de la cibercultura». Revista Mexicana de Comunicación, 23 (123), 24-26.

- (2011). «Comunicación, vida y sociedad. La sociogenética de la Teoría de la Comunicación frente a la paleontologenética de la Comunicación Humana». Chasqui: Revista Latinoamericana de Comunicación, 114-115, 66-68.

GARCÍA, A. (ed.) (2012). Comunicación, infancia y juventud: Situación e investigación en España. Barcelona: Editorial UOC.

GARCÍA CANCLINI, N. (1990). Culturas hibridas: estrategias para entrar y salir de la modernidad. México: Grijalbo, Consejo Nacional para la Cultura y las Artes.

Giraldo-Luque, S.; Tejedor, S. y CARniel-BugS, R. (2017). «Motivaciones de uso de las redes sociales de los estudiantes de periodismo de América Latina y España». Informação \& Sociedade: Estudos, 27 (3), 191-203. <https://doi.org/10.22478/ufpb.1809-4783.2017v27n3.34691>

GÓMEZ, J. (1990). «Metodología de encuestas por muestreo». En: J. ARNAU, M. T. ANGERA y J. GÓMEZ (eds.). Metodología de la investigación en ciencias del comportamiento. Murcia: Universidad de Murcia.

Holsti, O. R. (1969). Content Analysis for the Social Sciences and Humanities. Massachusetts: Addison-Wesley.

INTAL; LATINOBARÓMETRO (2016). Latinobarómetro 2016. <http://intal-alianzalb. iadb.org> [Fecha de consulta: 11/09/2017]. 
JENKInS, H. (2008). Convergence Culture. La cultura de la convergencia de los medios de comunicación. Barcelona: Paidós.

Jones, C.; Ramanau, R.; Cross, S. y Healing, G. (2010). «Net generation or Digital Natives: Is there a distinct new generation entering university?». Computers \& Education, 54 (3), 722-732.

$<$ https://doi.org/10.1016/j.compedu.2009.09.022>

LATAMCLICK (2017). Estadisticas de Instagram 2017 (América Latina). <https:// www.latamclick.com/estadisticas-de-instagram-2017> [Fecha de consulta: 04/12/2017].

LÉvY, P. (2007). Cibercultura: la cultura de la sociedad digital. Barcelona: Anthropos. - (1999). ¿Qué es lo virtual? Barcelona: Paidós Ibérica.

MANOVICH, L. (2005). El lenguaje de los nuevos medios de comunicación: la imagen en la era digital. Barcelona: Paidós.

MARTIN SERRANO, M. (2008). La mediación social. Madrid: Akal.

MARTÍN-BARBERO, J. (2015). «¿Desde dónde pensamos la comunicación hoy?». Chasqui: Revista Latinoamericana de Comunicación, 128, 13-29.

- (2009). "Cuando la tecnología deja de ser una ayuda didáctica para convertirse en mediación cultural». Education in the Knowledge Society (EKS), 10 (1), 19-31.

MCCRINDle, M. y WOlFIngER, E. (2010). The ABC of XYZ: Understanding the Global Generations. Sydney: UNSW Press.

PARISER, E. (2011). The filter bubble: What the Internet is hiding from you. Londres: Penguin.

Pérez Tornero, J. M. (2016). Promoting Digital Literacy. Informe Final EAC/76/03. Comprender la alfabetización digital. Barcelona: Gabinete de Comunicación y Educación, UAB. <http://www.gabinetecomunicacionyeducacion. $\mathrm{com} /$ sites/default/files/field/adjuntos/comprender_dl.pdf> [Fecha de consulta: $11 / 06 / 2017]$.

PÉREZ TORNERO, J. M. y TEJEDOR, S. (eds.) (2016). Ideas para aprender a aprender. Manual de innovación educativa y tecnología. Barcelona: Editorial UOC.

Piscitelli, A. (2002). Ciberculturas 2.0 en la era de las máquinas inteligentes. Barcelona: Paidós Ibérica.

Piscitelli, A.; Adaime, I. y Binder, I. (comp.) (2010). El Proyecto Facebook y la posuniversidad. Sistemas operativos sociales y entornos abiertos de aprendizaje. <http://www.codajic.org/sites/www.codajic.org/files/El\%20Proyecto\%20Facebook.pdf> [Fecha de consulta: 11/06/2017].

Postman, N. (1970). "The Reformed English Curriculum». En: A. C. EURICH (ed.). High School 1980: The Shape of the Future in American Secondary Education. Nueva York: Pitman, 160-168.

Roblyer, M.; MCDANiel, M.; WebB, M.; Herman, J. y WitTy, J. (2010). «Findings on Facebook in higher education: A comparison of college faculty and student uses and perceptions of social networking sites». The Internet and Higher Education, 3 (13), 134-140.

<http://dx.doi.org/10.1016/j.iheduc.2010.03.002>

Salaverría, R. (2016). "Redefinir al comunicador». El Profesional de la Información, 25 (2), 163-167. $<$ https://doi.org/10.3145/epi.2016.mar.02>

SAN JOSÉ, J. (2017). «Edad mínima para estar en las redes sociales». Derecho en la Red. <https://derechodelared.com/edad-minima-para-estar-en-las-redes-sociales/> [Fecha de consulta: 04/12/2017]. 
SCOLARI, C. A. (2013). «Media evolution. Emergence, dominance, survival and extinction in the media ecology». International Journal of Communication, 7 , 1418-1441.

- (2012). "Media ecology: exploring the metaphor to expand the theory». Communication Theory, 22 (2), 204-225. <http://dx.doi.org/10.1111/j.1468-2885.2012.01404.x>

SILVA, B. et al. (2014). «Aplicação e uso de Tecnologias digitais pelos professores do ensino superior no Brasil e Portugal». Educação, Formação \& Tecnologias, 7 (1), 3-18.

Singh, J.; Grizzle, A.; JoAN YeE, S. y Hope Culver, S. (eds.) (2015). Media and Information Literacy for the Sustainable Development Goals. MILID Yearbook 2015. Gothenburg: The International Clearinghouse on Children, Youth and Media; Nordicom; University of Gothenburg. <http://www.nordicom.gu.se/ sites/default/files/publikationer-hela-pdf/milid_yearbook_2015.pdf> [Fecha de consulta: 10/10/2017].

TECNÓSFERA (2015). «El 23\% de la población en Latinoamérica usa 'apps' de mensajería». El Tiempo. <http://www.eltiempo.com/archivo/documento/CMS16447337> [Fecha de consulta: 26/11/2017].

Toffler, A. (1980). The Third Wave. EE. UU.: Bantam Books.

TRAmullas, J. (2016). «Hannibal ad portas, o los futuros perfiles profesionales de la información». El Profesional de la Información, 25 (2), 157-162. $<$ https://doi.org/10.3145/epi.2016.mar.01>

Wolton, D. (2000). Internet, ¿y después? Una teoría critica de los nuevos medios de comunicación. Barcelona: Gedisa. 
